University of Massachusetts Amherst

ScholarWorks@UMass Amherst

Chemical Engineering Faculty Publication

Series

2011

\title{
Bio-Inspired Materials For Parsing Matrix Physicochemical Control Of Cell Migration: A Review
}

Hyung-do Kim

Massachusetts Institute of Technology

Shelly Peyton

University of Massachusetts Amherst

Follow this and additional works at: https://scholarworks.umass.edu/che_faculty_pubs

\section{Recommended Citation}

Kim, Hyung-do and Peyton, Shelly, "Bio-Inspired Materials For Parsing Matrix Physicochemical Control Of Cell Migration: A Review" (2011). Integrative Biology. 877.

https://doi.org/10.1039/c1ib00069a

This Article is brought to you for free and open access by the Chemical Engineering at ScholarWorks@UMass Amherst. It has been accepted for inclusion in Chemical Engineering Faculty Publication Series by an authorized administrator of ScholarWorks@UMass Amherst. For more information, please contact scholarworks@library.umass.edu. 


\title{
Bio-Inspired Materials For Parsing Matrix Physicochemical Control Of Cell Migration
}

\author{
A Review \\ Hyung-Do Kim ${ }^{1}$, Shelly R. Peyton ${ }^{2}$ \\ 1. Department of Biological Engineering, Massachusetts Institute of Technology, \\ Cambridge, MA \\ 2. Department of Chemical Engineering, University of Massachusetts, Amherst, MA
}




\section{ABSTRACT}

Cell motility is ubiquitous in both normal and pathophysiological processes. It is a complex biophysical response elicited via the integration of diverse extracellular physicochemical cues. The extracellular matrix directs cell motility via gradients in morphogens (a.k.a. chemotaxis), adhesive proteins (haptotaxis), and stiffness (durotaxis). Three-dimensional geometrical and proteolytic cues also constitute key regulators of motility. Therefore, cells process a variety of physicochemical signals simultaneously, while making informed decisions about migration via intracellular processing. Over the last few decades, bioengineers have created and refined natural and synthetic in vitro platforms in an attempt to isolate these extracellular cues and tease out how cells are able to translate this complex array of dynamic biochemical and biophysical features into functional motility. Here, we review how biomaterials have played a key role in the development of these types of model systems, and how recent advances in engineered materials have significantly contributed to our current understanding of the mechanisms of cell migration. 


\section{INTRODUCTION}

Human physiology, pathophysiology, and regenerative medicine are each built on the careful orchestration of cellular motile machinery. Embryonic morphogenesis relies on migratory events during gastrulation and neural crest development [1-3]. In vertebrate adults, cell motility is a critical part of wound healing and tissue repair [4]. Collective motility of epithelial cells constantly renews skin and intestinal tissue. Finally, immune surveillance would be impossible without the unique migratory ability of lymphocytes $[5,6]$. Dysregulation of migratory processes results in disastrous consequences, such as vascular disease, osteoporosis, chronic inflammatory diseases, multiple sclerosis, and even mental retardation. Aberrant cell migration in cancer (metastasis), is the leading cause of cancer-related deaths [7].

Therefore, understanding cell migration's role in healthy tissue, in disease progression, and for tissue engineering, has been of great interest to the scientific community. The elucidation of the signaling pathways underlying the regulation of motility has led to the identification of intracellular components that robustly respond to cues from the microenvironment, such as growth factors, cytokines, and physical cues from the extracellular matrix. Cell migration is a cycle of biophysical processes that are spatio-temporally regulated [8,9]. The cycle is initiated by the cell's extension of protrusions, which requires polymerization of actin microfilaments, aided by actinbinding proteins, such as Arp2/3, profilin, cofilin, and Ena/VASP proteins [10]. Protrusions at the leading edge of a cell (wide lamellipodia or finger-like filopodia) form stable adhesions to the ECM ligands via transmembrane proteins, called integrins [11, 12]. Protrusions and adhesions, often in conjunction with extracellular soluble gradients, establish an intracellular polarity, and activate a myriad of known and unknown signaling proteins [13] including the Rho-family GTPases [14-17]. Upon adhesion, active GTPases initiate a cascade of events, and myosin II generates forces by pulling on the actin microfilament network to translocate the polarized cell body [18]. The carefully regulated, spatio-temporally controlled activation of this signaling network also leads to the disassembly of adhesions at the trailing edge of the cell, and a net contractile force at the leading edge, allowing the cell to migrate [19]. Depending on the 
cell type, the orchestration of these processes can vary widely. The components of the migration cycle: protrusion, attachment, and contraction, can be observed as separate processes in mesenchymal-like cells, such as fibroblasts, whereas in amoeboid-like cells, such as leukocytes, different mechanisms of migration have been proposed [20, 21], and individual processes may be less easily discernable.

The resulting cell movement must occur in balance with the physical properties of the extracellular microenvironment. The ECM consists of various filamentous, amorphous, and cross-linking proteins, such as collagens, laminins, fibronectins, glycosaminoglycans, etc., and provides both a physical support and barrier for cell migration [22]. Many cells, therefore, have a framework for remodeling the ECM - from cleavage of ECM proteins through various secreted proteases to secretion of ECM proteins - and rely heavily on this framework for productive locomotion [23]. In contrast, recent work indicates that leukocytes do not require ECM modifying abilities and employ a proteolysis-independent mechanism to enable fast movement without destruction to the tissue [20,24].

The diversity of biophysical processes in cell migration has provided a great opportunity for the field of biomaterials to pose biologically relevant questions via the creative design of well-defined microenvironments. To date, much of the mechanistic research in the field has been generated in a physiologically inappropriate context: tissue culture plastic. Tissues in the human body are not two-dimensional, and have a far more complex physical and chemical microenvironment that dictates cell behavior. Further, two-dimensional studies naturally neglect proteolysis-driven motility and tissue invasion [25]. The biosciences community has increasingly sought more physiologically relevant systems, which permit the ability to quantitatively probe various aspects of cell migration in a diverse set of microenvironments. The biomaterials field has emerged out of a traditional landscape of inert material design into the engineering of bioinstructive, -specific, and -responsive tools over the last twenty years. Both tissuederived and synthetic materials can be rendered biologically sensitive and directive with the incorporation of adhesive matrix factors, signal-initiating growth factors (and growth factor depots), and enzymatic recognition sites (for review see [26]). New technology is continuously emerging to decipher how physicochemical cues from the extracellular 
matrix, such as chemical, nanotopographical, mechanical, and enzymatic cues, can feedback and regulate intracellular processes to study how cells make decisions about migration. Cell migration studies using these engineered materials have provided insights into a phenomenon that compels a quantitative approach for establishing relationships between migratory parameters.

In this review, we examine the use of biomaterials in elucidating novel insights into the mechanisms of cell migration, and attempt to place these findings into a physicochemical perspective. We outline our appreciation for the contribution of the current biomaterials literature to the general biochemical and biophysical understanding of cell migration. We specifically focus on the use of biomaterials to control chemotaxis, matrix adhesivity and haptokinesis/-taxis, matrix elasticity and durotaxis, cell polarity, and proteolysis, and the ability of these materials to allow discovery of key mechanistic parameters cells rely on to make decisions about cell migration in general. For literature describing cell migration relevant to specific physiology and pathophysiology, we refer the audience to other reviews [1-7, 9, 27]. 


\section{CHEMOTAXIS}

Chemotaxis is the directed migration of cells in response to a soluble chemical signal. Chemotaxis-driven migration is prevalent in many biological events, such as development, the immune response, and wound healing. For example, interstitial flow induces a gradient of chemokines that directs cell migration for lymphatic angiogenesis [28-30]. The release of soluble factors from leaky vessels and macrophages is common in damaged tissues and disease states, eliciting unwanted migration and disastrous consequences, such as in cardiovascular disease or metastasis. For example, a mutual chemotactic gradient established between macrophages and carcinoma cells through paracrine release of EGF and CSF-1 enhances tumor metastasis [31, 32].

In vitro assays to study chemotaxis have been under development for the past three decades, beginning with simple studies of cells migrating on glass surfaces in the presence of localized soluble factors. The Boyden migration chamber was the most notable early advancement in tools development to study cell invasion toward chemoattractive factors [33], where cell invasion is measured through an adhesive porous filter toward a chemoattractive factor. No matrix degradation is required for invasion, but pore sizes of the filter can be varied to assess the effect of steric barrier. The under-agarose assay was the first biomaterials development to add a 3D context to better mimic an in vivo environment for chemotactic studies [34]. In this assay, chemoattractants are deposited into a small well within a 3D agarose gel, while a suspension of cells is placed nearby. Cells sense and migrate toward the diffusive gradient of soluble factors through the nanoporous hydrogel. This assay can recapitulate some of the physiological aspects of chemotactic responses important in inflammation. Though quite simple, relevant quantification of diffusion characteristics, as well as visualization of cell motility can be achieved from this assay [35].

Both the under-agarose and Boyden chamber are end-point assays, prohibiting assessment of individual cell kinetics. In addition, because these assays use a single sink of soluble factor, chemoattractants do not form stable gradients, as is often observed in physiological conditions. The development of microfluidic devices to create stable and reproducible gradients of chemical factors has produced chemotaxic 
microenvironments closer to physiologic conditions than ever before achievable. Surprisingly, very basic microfluidic systems have been around and used for short timepoint bacterial migration assays for many years [36]. More recently, microfluidic platforms have evolved to include 3D gels, overlaid gradients of multiple factors, and separated chambers to analyze cell-cell communication and coordinated motility. Microfluidic platforms are now commercially available to study the migratory response of immune cells to antibodies, shear stresses, and inflammatory cytokines [37].

Microfluidic platforms have made quantitative 3D models of real-time chemotaxis possible, both for adherent and suspension cells [38]. Using a 3D platform that separated cell-seeded areas and gel-only areas, Chung et al. observed cancer cell migration through 3D materials toward a stable gradient of VEGF [39], recapitulating invasion during metastasis in the presence of leaky vasculature. This device also allows for sophisticated co-cultures of endothelial cells, smooth muscle cells and cancer cells to investigate the coordinated cell-cell communication, as one would find in a wellvascularized tumor microenvironment. The authors observed that cancer cells promoted endothelial migration (to different degrees, depending on the cancer cell subtype), while the presence of smooth muscle cells inhibited endothelial motility.

Chemotactic motility in the presence of multiple growth factors is possible with ladder chamber microfluidics, which include multiple compartments, and generate stable diffusion in both 2D and 3D geometries, in the absence of shear flow (a common component of other microfluidics platforms, which can influence cell behavior in an uncontrollable fashion) [40]. With this platform, researchers generated a stable gradient of IL-8, and directed the migration of neutrophils. By being able to tightly control shear forces and chemical gradients independently, these types of systems could lend powerful insight in the study of neutrophils responding to inflammatory cues, as well as cancer cell motility toward leaky vessels in physiologically relevant 3D microenvironments.

The studies thus far have all discussed the diffusion of soluble factors through a media solution or semi-porous 3D milieu. However, growth factors in vivo are commonly found covalently, ionically, or physically connected to the surrounding insoluble fibrous matrix. Advances in wet surface chemistries have enabled creation of 
stable gradients of growth factors tethered to 2D surfaces to study the interaction of cells with covalently bound chemokines. The biomaterials community has found chemical methods to tether growth factors to $3 \mathrm{D}$ systems without interfering with their bioactivity [41-43]. For example, recent work by Stefonek-Puccinelli and Masters exploited surface chemistry techniques to overlay gradients of EGF and IGF-1 onto standard tissue culture plates [44]. Subtle effects were seen when comparing the growth factors, but they observed a dramatic increase in overall kerotinocyte migration by covalently linking growth factors to the surface of the plates when comparing to the growth factor free control. In addition, they observed a biphasic relationship between cell migration and maximum EGF concentration, with an optimal migration at an intermediate concentration of EGF, which has also been described for chemotaxis with soluble growth factors [45].

Biomaterials systems have enhanced the study of chemotaxis in a myriad of ways. Unlike glass and plastic substrata, biomaterials have large pore sizes (on the order of nanometers), which allow for diffusion of chemoattractants or flow rates of fluids. Pore sizes can be easily tuned by varying the crosslinking densities of the biomaterial. The addition of interstitial flows in controlled environments can be used to study combinatorial effects of chemical and mechanical factors that are physiologically relevant to lymph draining and tumor cell intravasation [46]. These types of systems may better mimic the interactions that cell surface receptors have with extracellular factors in the body, leading to interesting discoveries. 


\section{CELL ADHESION AND HAPTOTAXIS}

In addition to soluble factors, cell motility is also regulated by insoluble adhesive matrix components. Adhesive protein-mediated motility is called haptokinesis, while directed motility by an adhesive gradient is called haptotaxis. Adhesive domains in the ECM not only serve as a physical mechanism for cell adhesion, but also as complex biochemical regulators of a variety of cellular responses, including motility $[47,48]$. The diversity of ECM composition in various tissues indicates that, like soluble chemokines, levels and distribution of Type I and IV Collagen, fibronectin, and laminins have a profound effect on cell migration responses. The dynamic assembly of focal adhesions, a cluster of proteins connecting integrins to the intracellular cytoskeleton, as observed in two-dimensional (2D) and three-dimensional (3D) cultures, plays an important role in cell migration [47, 49, 50]. Therefore, many efforts have been directed towards quantifying the contribution of adhesions to the overall migratory behavior by controlling the microenvironment in which adhesions are formed [51, 52].

Nearly fifteen years ago, $\mathrm{CHO}$ cell and smooth muscle cells were shown to have a biphasic cell migration speed dependence on the concentration of insoluble adhesive proteins passively adsorbed on glass slides $[53,54]$. Simple control of ECM ligand density was achieved via adsorption coating of glass slides with purified natural ECM proteins, ranging from fibronectin, collagen, laminin, or fibrinogen. The observed biphasic dependence could be further tuned by knocking down integrin expression targeting the ECM proteins. Ten years later in 3D microenvironments, modulation of PtK1 epithelial cell migration under dose-dependent inhibition of myosin [55] was consistent with an ECM ligand density-dependent increase in fibroblast cell speed on fibronectin when stimulated with EGF [56]. There also existed an optimal fibronectin concentration, at which EGF stimulation resulted in the greatest increase in cell speed. Thus, EGF stimulation, through its activation of various contractility pathways including ROCK [57], could provide a compensating increase in cell contractility, but could only demonstrate optimal compensation at a particular fibronectin concentration. At very low concentrations of fibronectin, EGF stimulation decreased cell speed indicating that the balance-disrupting increase in contractility could result in a reduction in motility [56]. 
These studies demonstrated that systematic variation of physicochemical cues is vital to deconvolve the complex biophysical processes involved in cell migration.

The conjugation of adhesion sites into biomaterials has been of great interest to the community to optimize cell colonization [52]. A variety of polymers have been conjugated with either natural proteins, the IKVAV peptide present in laminin [58], or, most prominently, the RGD peptide, which is present in many ECM proteins, and recognized by multiple integrin subtypes [59]. For example, in conjunction with sphingosine-1-phosphate $(\mathrm{S} 1 \mathrm{P})$, researchers at Washington University conjugated varying ratios of synthesized RGD (or cyclic $R G D$ ) and poly(ethylene glycol) (PEG)-vinyl sulfone hydrogel precursors [60]. They confirmed previous reports that endothelial cell migration on RGD-containing hydrogel depended biphasically on RGD concentration. Importantly, this biphasic curve shifted in the presence of S1P, demonstrating the interplay between soluble and insoluble cues in regulating motility. The biphasic dependence of cell speed has also been demonstrated in numerous 3D constructs including in prostate cancer migration in Matrigel [61], and both fibrosarcoma and smooth muscle cell migration in PEG-based gels [62, 63].

The combination of cell speed and directional persistence determines the total distance travelled for a single cell [64]. The significance of directional persistence during haptokinesis is not fully understood; however, parsing the effects of various migratory phenomena, such as haptokinesis, haptotaxis, chemokinesis, and chemotaxis, will aid in predicting the integration of all these effects during physiologically relevant cell migration. Interestingly, NR6 fibroblasts exhibit directional persistence that is highest at an intermediate Amgel coating concentration [65]. However, stimulation with a bath application of EGF decreases directionally persistent migration $[65,66]$. The authors claim that EGF may increase cell speed in metastatic cells, but decrease directional persistence in order to increase the total area probed via randomly directed environment sensing. However, this biphasic directionality response has not been universally reported. In the study on S1P, persistence is only weakly dependent on RGD ligand density, and only subtly responsive to S1P stimulation [60]. Therefore, it appears that cell persistent migration is not necessarily correlated with cell 
migration speed. Future rational biomaterial design will likely require fine-tuning over multiple independent physical parameters to maximize directional cell migration.

A unique area of impact provided by the biomaterials community has been the nano-scale control and presentation of biochemical cues. For example, Spatz et al. initially demonstrated that by allowing di-block copolymer micelles containing gold nanoparticles to form on glass substrates, they are able to obtain well-controlled spacing of gold particles [67]. Careful variation of the copolymer concentration achieves particle spacing between $28 \mathrm{~nm}$ and $110 \mathrm{~nm}$. Subsequent conjugation of gold nanoparticles with thiol-conjugated cyclic RGD peptides resulted in well-defined separation of RGD adhesion sites, in which only one integrin theoretically binds to the adhesion site. This is in sharp contrast to varying bulk ligand density, in which ligand presentation is ultimately stochastic. On surfaces with large RGD spacing, rat fibroblasts are unable to spread properly due to lack of focal adhesion stability [67]. Fibroblasts on large RGD spacing migrate quicker, but with less persistence, perhaps due to the lack of cell spreading and its influence on polarity. It has been previously demonstrated that fast turnover in focal adhesions, or the short-lived focal contacts, is correlated with increased cell migration speed $[68,69]$. Spatz et al. also used the gold nanoparticle approach to create well-defined linear gradients of RGD spacing, via controlled retraction of the substrate from the copolymer solution during coating [70]. Here, the authors show that cells polarize and exhibit tendency to migrate towards the higher gradient of adhesive ligand. While single RGD peptides were presented in the above studies, others have used controlled substrates to study the effect of integrin clustering on cell motility [56]. To do this, RGD was linked to a star-shaped polymer consisting of PEG linkers and conjugated to a PEG hydrogel. Clustered spatial organization of RGD increased NR6 fibroblast migration speeds and robust stress fiber formation compared to unclustered RGDs. This type of controlled spatial presentation of ligands is physiologically relevant, as the in vivo ECM likely presents an unpredicted spatial presentation of integrin binding sites as well as bound growth factors.

As stated earlier, physiological migration does not occur on substrates with homogeneously distributed adhesion sites. The cell migration microenvironment consists of varying levels of ECM components with localized gradients. Therefore, 
great interest exists in understanding the cell's migratory response upon encountering haptotactic cues. There are various biomaterial technologies suited to create molecular gradients, including self-assembled monolayers (SAMs) and PEG hydrogels $[51,62,71$, 72], which can be combined with microfluidic systems [73] to enhance local cell motility. SAMs are generally created via assembly of alkane-thiols onto gold surfaces and subsequent biomolecule conjugation. Migration of bovine aortic endothelial cells on fibronectin gradients is directed distinctly towards increasing adhesion sites [74, 75]. Not surprisingly, the haptotactic effect can be enhanced by growth factors [76]. RGD gradients in PEG gels can be created by UV polymerizing RGD-conjugated PEG diacrylate and unconjugated PEG diacrylate at different concentrations [77], or by polymerization of microfluidics-mediated RGD-PEG precursors [78]. Both human dermal fibroblasts and mouse embryonic fibroblasts in these studies, respectively, enhanced their migration speeds towards increasing RGD density, and in both cases, migration speed was dependent on the slope of the gradient. Combination of contact printing and SAM can print ligands to different regions to control cell adhesion [79-81]. These types of technologies, impossible without the integration of biological insight and biomaterials engineering, show promise in regulating cell migration, both in the context of future rational scaffold design and for mechanistic understanding. 


\section{MATRIX MECHANICS AND DUROTAXIS}

It is now widely accepted that mechanical forces from the extracellular matrix play a large role in directing tissue morphogenesis and progenitor cell lineage commitment, where mechanical forces can be transferred between cells through cadherins and the intermediate filaments, or between cells and the matrix through focal adhesions and the actin cytoskeleton. A subset of mature tissues experience dynamic mechanical loading in vivo, such as in the cardiovascular and musculoskeletal systems, and these mechanical forces are thought to be critical for the maintenance of proper smooth muscle and bone cell phenotype. Also, endothelial cell function is regulated by the constant shear stresses conferred to them in cardiovascular and pulmonary tissues. In fact, when cells from these tissues are removed from the body and cultured on tissue culture plastic, they markedly lose proper differentiation marker expression, and convert to a synthetic phenotype. Mechanical forces (both static and dynamic) from the ECM in vitro are known to influence endothelial cell behavior (for review, see [82]), stem cell lineage commitment [83], both smooth and skeletal muscle cell plasticity [84-86], osteoblast phenotype [87], as well as many other cell and tissue behaviors (for review, see [88]).

The first evidence that the static mechanical properties of the ECM could be translated into functional changes in migratory phenotype came from Yu-Li Wang's lab over a decade ago. They created engineered substrates from polyacrylamide (PAA) hydrogels, more traditionally known for their tunable mesh sizes, and used in molecular biology for protein separation [89]. Although disputed by some [90], these substrates were reportedly the first of their kind to demonstrate independent control over substrate stiffness and the concentration of adhesive ligand presented at the surface. These tunable mechanical properties were exploited to create a 2D substrate that contained an interface between a compliant and a stiff surface, with the integrin-binding collagen protein covalently linked to the surface to facilitate cell adhesion. When 3T3 fibroblast migration was tracked using time-lapse microscopy, cells initially adhered on the soft gel were found to preferentially migrate onto the stiffer surface. Conversely, cells initially adhered to the stiffer surface would preferentially stay on the stiffer substrate (Figure 
3A-B). This first evidence of stiffness-directed cell motility is now widely known as mechanotaxis, or durotaxis.

In a more quantitative study, human aortic smooth muscle cell migration was compared on uniform PAA substrates of different stiffnesses [91]. Similar to variation of adhesivity, cells migrated fastest on substrates of intermediate stiffness and exhibited a biphasic migration speed as a function of static matrix stiffness (Figure $3 \mathrm{C}$ ). This experiment corroborates that force balance between ECM tension and cytoskeletal contractility is critical in generating motility. Indeed, the stiffness value at which this maximum migration speed occurred depended on the concentration of adhesive protein presented at the surface. This biphasic phenomenon has also been seen with both osteoblasts [92] and neutrophils [93], but not necessarily with all cell types [94], suggesting a differential role of contractility in cell migration across cell types. Nevertheless, these studies provide a mechanistic foundation for the durotactic behavior observed in Yu-Li Wang's study.

The development of stable gradients of stiffness within 2D substrates has been a powerful advancement in the study of durotaxis phenomena. PAA substrates can be created with a gradient in stiffness with a simple, yet elegant technique of combining photo-masking with photo-sensitive polymerization [95]. On these surfaces with gradients in stiffness, vascular smooth muscle cells (SMCs) migrated radially from softto-stiff regions of the hydrogel. Using a sophisticated microfluidics approach, Burdick et al. created gradients in both the concentration of adhesive peptide (RGDS) and PEG crosslinker content [96]. Though not fully explored within this study, systems such as these could be used to study the cross talk of adhesive and stiffness cues in the directed migration of cells in 2D. Expanding on this microfluidics approach, Zaari et al. created gradients in stiffness ranging from $\sim 1$ to $40 \mathrm{kPa}$ on a single PAA gel within a $3 \mathrm{~mm}$ total distance [97]. In agreement with this lab's previous work, an increase in SMC attachment, total spread area, and F-actin fiber definition was observed with increasing substrate stiffness along the gradient. It was not clear from this study, however, if cells were able to migrate preferentially toward the stiff region of the gel, or if the increase in cell number on the stiffer regions was due to an increase in attachment. Isenberg et al. followed up this study using the same microfluidic platform to discern 
whether or not a gradient in stiffness could direct SMC migration [98]. They found that SMCs were responsive to the stiffness cue, and that the strength of the gradient controlled how responsive cells were to the stiffness cue given. Presumably, if a stiffness gradient is too gradual, changes in stiffness will not occur within the same length scale of the cell. Unlike soluble growth factors, this stiffness cue is static, and a cell may have to encounter the cue probabilistically during its random walk for it to have an effect.

Mechanosensing in 3D model systems is just beginning to be investigated. To parse the roles of 3D matrix mechanics and cell motility, a number of natural biopolymers have been widely embraced, especially Type I Collagen [99] and Matrigel. These systems offer an extreme ease of use, as they can be commercially purchased, contain natural cell-adhesive domains, are enzymatically degradable, and can be made to span a small range of stiffnesses. Using a nested collagen matrix technique pioneered by Fred Grinnell, Miron-Mendoza et al. showed that the stiffness of the outer cell-free matrix increased the ability of human foreskin fibroblasts to migrate [100]. When the outer matrix was soft, cell migration slowed, and it was possible to visualize collagen fibers moving under cell tractional forces. They hypothesized that motile cells use tractional forces to pull on collagen fibrils to move forward, so when the outer matrix is restrained by internal crosslinks, cell tractional forces can be completely transduced into cell motility. In lieu of increasing crosslinker content, cell migration was also maximized by constraining the outer gel onto the walls of the polystyrene tissue culture plate.

Using Matrigel as a model system, Zaman et al. reproduced the biphasic dependence of migration speed on material stiffness in human prostate carcinoma cells (Figure 3D) [61]. Interestingly in this 3D environment, the relationship between cell migration speed, matrix stiffness, and matrix adhesion did show the same curve shift as was previously shown on 2D substrates [91]. As the 3D environment was softened, cell migration speed was maximized in conditions with lower adhesivity. At first glance, this result contradicts previous work on 2D substrata. However, many parameters require a closer look in this Matrigel study, including the difference in cell types studied (highly invasive prostate carcinoma cells vs. the generally less-motile primary, fully 
differentiated SMCs), and the difference in the stiffness ranges tested. The authors make many suggestions as well, including the added viscoelastic resistance of a 3D matrix of entangled fibers affects the ability of cells to polarize, which may account for this difference in balance of adhesive and mechanical traction forces, forcing cells to alter their morphology to squeeze through pores. The authors compared these 3D relationships between stiffness, adhesion, and proteolysis in a parallel computational model [101]. However, the lack of control of physicochemical properties in this and other protein-based 3D systems may produce convoluting factors. Matrigel concentration is increased to stiffen the resulting $3 \mathrm{D}$ gel, which also alters the adhesive ligand concentration, pore diameters, diffusion of growth factors, and the availability of enzymatically-sensitive peptide domains.

One challenge ahead is to elucidate the mechanism of force transduction. Canonically, it is theorized that mechanical signals from the ECM are transmitted to the internal structure of the cell via integrins (for review, see [102]). Several studies have pointed to integrins as providing a link between mechanical changes in the matrix and cell migratory phenotypes $[61,103,104]$. Further, focal adhesions are known to be sensitive to substrate mechanical properties [105, 106]. However, it is still not completely understood how integrins are able to transfer mechanical information between the cell and the ECM. One theory is that cells are constantly probing their mechanical environment and exerting tension via myosin contraction along F-actin filaments [107, 108]. A motor-clutch mechanism may explain how maximum migration speeds have been observed on substrates of intermediate stiffness, as intracellular tension increases with increasing substrate stiffness up to a point of frictional slippage [108]. The mechanisms behind which cells are able to respond to mechanical cues in 3D are less clear, as natural biopolymer systems (such as Type I Collagen and Matrigel), are unable to separate stiffness cues from proteolytic and adhesive cues. Current synthetic gel systems achieve this goal, but still have problems of convoluting stiffness and porosity/diffusion parameters. New technologies are on the verge of achieving this goal, including macroporous synthetic scaffolds [109], and 3D systems with gradients in elasticity [110]. 
Given the diversity of mechanical environments in vivo, the ability to engineer substrates that contain tunable static and dynamic mechanical properties is extremely useful. Further, the loss of these naturally occurring mechanical forces with tissue culture plastic dishes may be a key factor in why cells undergo unnatural changes in their phenotype in culture. Ideally, biomaterial tools will include tight control over all these physicochemical features, such as adhesivity, microarchitecture, and stiffness, so that engineers and biologists are able to study cell motility in more physiologically relevant environments, yet more reliably, reproducibly, and cost effectively than in animal models. 


\section{PROTEOLYSIS}

There is increasing interest in studying migration in 3D environments, with the awareness that the vast majority of migratory microenvironments in vivo are composed of cells surrounded by matrix in all dimensions [22, 111, 112]. The most researched 3D motility phenomenon is ECM proteolysis, specifically proteolysis mediated by members of the matrix metalloproteinase (MMP) family [113-115]. Cell migration in 3D ECM is complicated by the existence of steric barriers, created by the integration of a diverse profile of insoluble scaffolding proteins, such as fibrous, porous Type I Collagen networks and the denser, amorphous laminin-fibronectin networks [48]. To overcome steric barriers, cells possess an arsenal of MMPs, as well as proteolysis-independent motility mechanisms [21]. The secretion of MMPs and their inhibitors, is, like all cellular processes, a tightly regulated process stimulated by a variety of physicochemical cues [113]. Biophysically, matrix remodeling results in local and global changes in ligand density, matrix stiffness, and scaffold geometry, which feedback to regulate cell motility. Therefore, proteolysis-mediated cell migration poses an interesting feedback mechanism that still requires elucidating for a variety of purposes, including understanding tumor invasion and the rational design of tissue engineering constructs. Controlled microenvironments provide an opportunity to deconvolve the complexity involved in proteolysis-mediated cell migration.

As an extension to the previous computational model addressing elasticity and adhesivity in 3D cell migration [101], Zaman et al. incorporated the effects of matrix porosity and MMP activity in a lattice Monte-Carlo model [116]. Not surprisingly, the model predicted a non-linear dependence of cell speed on ligand density. However, due to the advantages of the modeling technique, it also predicted that directional persistence depended biphasically on ligand density. In their model, persistent migration is enhanced in the presence of MMP activity; however, the increase is less dramatic if the model incorporates the cell's ability to deform the matrix and decrease local steric hindrance. While mesenchymal-type migration in physiology involves both matrix deformation and proteolysis [115], this study conceptualizes the effects of distinct matrix remodeling processes involved during 3D cell migration. 
Experimentally, in one of the earliest reported, controlled 3D motility studies, RGD peptides were conjugated into Type I Collagen matrices, which are naturally degradable [117]. Mouse melanoma cells moving in these matrices exhibited a biphasic dependence of directional persistence on RGD concentration. Secondly, in a study utilizing a novel engineered PEG-based hydrogel containing MMP degradation sites, fibroblasts enhanced MMP release upon TNF- $\alpha$ stimulation, which increased the motility persistence length [118]. In the first study, gradually modulating cellular MMP activity via pharmacological inhibition, EGF-enhanced human glioblastoma cell migration in 3D collagen matrices was compared to 2D collagen-coated substrata [119]. Surprisingly, EGF stimulation increased directional persistence in 3D collagen, but decreased persistence on 2D collagen surfaces. By modulating MMP activity and matrix degradation with a broad MMP inhibitor, authors showed that the EGF-enhanced increase in directional persistence arises due to MMP activity, further corroborating hypotheses generated in the above computational model [116]. Interestingly, cell speed varied minimally with matrix degradation, which was consistent with the minimal dependence of cell speed on bulk collagen concentration. Collagenase itself has been shown to exhibit a persistent proteolysis along collagen fibrils [120], perhaps laying the molecular foundation for the high dependence of directional persistence on MMPmediated ECM degradation.

Most of the quantitative parametric studies involving proteolytic migration have been gathered on biopolymer matrices $[100,121]$. While physiologically relevant, they have the distinct disadvantage of convoluting matrix parameters, i.e. the number of available proteolysis sites is related to ligand density, matrix porosity, and bulk matrix stiffness. In response to this, a burgeoning number of studies have begun using synthetic materials, equipped with adhesive ligands and enzymatically-sensitive crosslinks [122]. PEG hydrogels have been particularly popular in this regard for their versatile chemistry and quantitative control. One of the first of such hydrogels incorporated integrin-binding peptides, the MT-1 MMP-sensitive peptide sequence GPQGIWGQ, and a plasmin-sensitive sequence YKNRD via multi-arm PEG monomers $[122,123]$. Subsequent studies have utilized step-growth polymerization of PEG monomers [124] or different functional end groups [63] to incorporate both adhesion and 
MMP-sensitive sequences, or even full length proteins [85]. Further, collagenasesensitive substrates have been incorporated into PEG hydrogels, allowing live visualization of collagenase activity during cell migration [125]. PEG hydrogels also have the ability to present a variety of receptor-binding biomolecules, with celldemanded release. In collaboration between the Langer and Hubbell groups, an MMPresponsive PEG hydrogel was used to encapsulate thymosin $\beta 4$, which induces vascular cell survival and upregulation of vascularization genes and MMP secretion, mimicking, perhaps, the release of matrix-associated growth factors in natural ECMs. Lastly, a recent report describes the use peptide-based hydrogels containing MMP2 recognition sites and RGD [126]. Parameterizing cell migration using synthetic ECMs is still scarce. However, due to the variety of ECM proteins with specificities for a diverse set of proteases, such studies would undoubtedly provide interesting insights into other mechanisms for regulating cell migration through presentation of diverse physicochemical cues.

Fueled by innovations in microscopy in the last ten years, interest in 3D cell migration has identified non-proteolytic forms of cell migration. The amoeboid mode of migration was first observed in fast-moving lymphocytes, and verified in vitro when fibrosarcoma cells were observed to undergo dramatic changes in morphology to allow for continued movement in 3D Type I Collagen gels in the presence of a cocktail of MMP and protease inhibitors. This seminal study provided a putative explanation for the poor success of MMP inhibitors as metastasis-targeted chemotherapy [127]. This mode of migration is integrin-independent, and utilizes a squeezing motion based on expansive actin network-based protrusive flowing and myosin-dependent contraction, which is required to pass the narrow gaps of ECM pores [20, 24]. While the mechanism holds water for lymphocytes, the mesenchymal-amoeboid transition of fibrosarcomas is currently disputed, due to the convoluting nature of pepsin-treated Type I Collagen. Ensuing studies in native Type I Collagen, which retain the natural crosslinks existent in vivo, were not able to replicate this MMP-independent motility [128]. These studies reiterate the importance of providing disease-relevant physicochemical cues. Synthetic PEG hydrogels incorporating both RGD and MMP-sensitive peptides, with inherent pore sizes orders of magnitude smaller than the smallest cell protrusion, have demonstrated 
that fibrosarcoma cells exhibited rounded and contraction-dependent migration, which is only weakly dependent on integrins [63]. While no proteolysis-specific measurements were performed, the authors suggest that fibrosarcoma cells in vivo are more rounded than they appear in traditional in vitro studies, and synthetic ECMs could provide a unique framework to examine these proposed mechanisms of motility and morphology. 


\section{MICROARCHITECTURE}

Several groups have highlighted the relevance of studying migration in 3D models due to the striking differences drawn between cells in 2D versus 3D matrices [129, 130]. Evidence suggests that both cell morphology and adhesion structure change dramatically between 2D and 3D cultures [50], perhaps due to the difference in structure between a monolayer of adhesive proteins on a flat surface versus the fibrous presentation of adhesive sites in 3D. In a study using the same matrix protein, research has shown that migration speeds on $2 \mathrm{D}$ collagen-coated surfaces do not correlate with speeds in 3D collagen gels, and that protrusion activity is controlled by distinct focal adhesion proteins ( 130 Cas in $2 \mathrm{D}$ and zyxin in $3 \mathrm{D}$ ) in the geometrically distinct microenvironments [131].

From an engineering perspective, although interesting, this study and others attempting to decipher the role of geometry on cell behavior separately from other biophysical factors are limited by the fact that these parameters are convoluted in natural biopolymers, such as Type I Collagen gels. This is highlighted when comparing the work of others in these gels, wherein the mode of matrix polymerization had profound affects on migratory phenotype (Figure 4) $[119,132,133]$. Work by Kim et al. observed that glioblastoma cells were insensitive to changes in $3 \mathrm{D}$ collagen stiffness and adhesivity [119]. However, Harley et al. showed that cell motility was dependent on the stiffness of collagen matrices that were formed using freeze-drying techniques [132]. This freeze-drying technique of matrix formation created pore sizes that were in excess of $100 \mu \mathrm{m}$ in diameter, so it is likely that cells in these matrices were experiencing a quasi-1D migratory microenvironment, rather than achieving true 3D migration.

To assess the effects of dimensional geometry on motility, Doyle and colleagues compared motility on a fibrillar cell-derived 3D matrix, flat PDMS model substrates, and PDMS printed lines [133]. They found that cell speeds along the PDMS lines ("1D mode") mimicked speeds along matrix fibers ("3D mode"), and that both of these morphologies led to speeds that were much faster than cells on uniform 2D surfaces. Work by Liu et al. implies that there is a minimal fiber diameter of $1 \mu \mathrm{m}$ to cause cells to polarize along the fiber [134]. Cell motile phenotype on fibers less than $1 \mu \mathrm{m}$ in diameter 
mimicked those on 2D surfaces. Most interestingly, the dependence of migration on ligand density, myosin, and microtubules was different between 1D and 2D migration, with 1D relationships most mimicking the 3D environment [133].

A recent study by Ochsner et al. has attempted to separate geometric influences from adhesive and stiffness effects by micropatterning different-shaped wells in PDMS substrata [135]. Their evidence suggests that actin filaments contribute to cytoskeletal tension, matrix remodeling, and metabolism differently on $2 \mathrm{D}$ surfaces versus $3 \mathrm{D}$ microenvironments. In fact, their study concluded that the geometric microenvironment determined the extent to which cells were sensitive to alterations in matrix stiffness by regulating cytoskeletal tension. Macroporous scaffolds made from inverse-opal processing are additional biomaterial tools capable of providing a nondegradable 3D environment with independently tunable adhesivity, stiffness, and pore diameters [136, 137]. Generally made with a PEG background, these scaffolds are inherently nondegradable, allowing for long-term cell tracking in a static system. Although not fibrillar, nor necessarily representative of in vivo tissues, these types of scaffolds are excellent model systems appropriate for parsing the relationships between cell motility and biophysical cues in 3D. Their macroporosity allows them to be overlaid with other degradable materials as well [138]. Recently, these PEG-based macroporous scaffolds were used to determine the effects of pore diameters on mesenchymal stem cell (MSC) motility [139]. Surprisingly, MSCs were observed to migrate in a non-intuitive fashion, where maximum displacement occurred in an intermediate pore diameter that was smaller than the spherical cell diameter, and maximum displacement did not correlate with maximum observed cell speeds. Cell speed had biphasic dependence on scaffold adhesivity, but only in environments that had very large pore sizes, and likely were a quasi-2D environment.

Thus far, we have discussed 3D synthetic systems that, although are more than ninety percent water, consist of a nanoporous mesh. These mesh sizes, resulting from crosslinking between polymer chains, are orders of magnitude smaller than the smallest cellular processes, and do not resemble the microarchitecture of an in vivo fibrous matrix (Figure 1). To create synthetic scaffolds that more closely mimic the native architecture and the nano- or micro-topology of the ECM, researchers have applied 
electrospinning techniques. Electrospinning has been in use for more than seven decades, but has only recently been employed to create synthetic tissue environments. Electrospinning can be used with a variety of polymer systems, and can create fibers with diameters ranging from $100 \mathrm{~nm}-10 \mu \mathrm{m}$, which spans the length scale of many natural fibers, such as collagens, chitosan, fibrin, chitin, and fibrinogen (for review, see [140]). Thus far, research with electrospun fibers has been focused on in vivo applications for tissue regeneration, but an electrospun polymer system could be used to study cell motility mechanisms in a synthetic polymer system with control of fiber architecture.

Self-assembled peptide gels (SAPGs) also form fibrillar matrices, and are able to mimic the geometrical features of in vivo collagen fibers. SAPGs are designed from natural amino acids and undergo spontaneous assembly into nanofibers, approximately $10 \mathrm{~nm}$ in diameter. Their pore sizes are on the order of hundreds of $\mathrm{nm}$, so cellmediated degradation is required for productive locomotion. Although the length scale features of these SAPGs are comparable to natural biopolymers, the motility of cells within them is markedly reduced in comparison [141]. Addition of osteogenic growth factor peptides into SAPGs can increase osteoblast motility alongside proliferation and expression of differentiation-specific markers [142]. Much smaller length scales have also been shown to be important for regulating cell responses to materials. Nonbiological $\mathrm{TiO}_{2}$ nanotubes have shown that cells are able to sense material properties on the order of nanometers, and have helped parse the relationship between protein spacing and cell migration in an inherently non-fouling environment. Focal adhesion formation and cytoskeletal assembly appears to be directly regulated by the nano-length scale and presentation of these nanotubes [143, 144], presumably mediated by adhesive matrix protein presentation.

Overall, it's clear from these studies that the architecture of the microenvironment plays a large role in directing cell motility. In vivo, varied microenvironments exist, including relatively constant 2D, planar basal laminas, largely porous, and potentially quasi-2D on the length scale of a cell, trabecular bone, fibrous and dense connective tissue, etc. Certain subtypes of cell populations dominate these environments, and these microarchitectures likely regulate the motility of these cells in vivo. Studies in 
which the microarchitecture of the $2 \mathrm{D}$ or $3 \mathrm{D}$ substrate can be controlled in vitro are therefore extremely relevant for the proper understanding and manipulation of the migratory behavior. 


\section{TOOL DEVELOPMENT}

Despite the many studies discussed within this review, the vast majority of cell motility studies are conducted on traditional glass or plastic surfaces. One of the reasons for the hesitation of the biological community to embrace biomaterial systems is the chemistry expertise needed (or perceived to be needed) to create biomaterial model systems versus the traditional platforms. Many scientists prefer naturally derived biopolymers, such as Type I Collagen and Matrigel, because although they have obvious reproducibility limitations, they are very easy to create, and their fibrous nature represents the in vivo microenvironment much better than the nonporous or macroporous 3D hydrogels described here. Certain technological and cost limitations also exist in imaging technologies to transfer mechanistic studies, canonically performed on thin, flat, and optically clear coverslips into more realistic 3D microenvironments. This section describes some of the more recent advances both within and outside the biomaterials community to overcome these hurdles.

Numerous biomaterial systems developed by the community have been created for tissue engineering purposes, but also show extensive promise as model substrates to study mechanisms of cell motility. For instance, because of their modular peptide design, protein-engineered biomaterials can mimic various properties of the natural ECM while maintaining the versatility to include non-naturally occurring binding sites for some synthetic "plug and play" control [145]. Though touted for their potential impact in the field of tissue regeneration, due to their natural bioresorbability and biofunctionality, these materials could also be useful for more basic mechanistic questions about cellmicroenvironment relationships due to the inherent molecular-level design control.

Confocal and two-photon lasers have taken advantage of UV-mediated polymerization of hydrogels to create 3D micropatterned materials (Figure $5 \mathrm{~A}$ ). Using this sophisticated form of photolithography (two-photon laser scanning, or TPLS), Lee et al. made 3D gels with precise control over the location of cell-adhesive RGDS in a degradable environment [146]. In doing so, they were able to guide the adhesion, and therefore migration, of fibroblasts in 3D. These types of 3D micropatterned gels may lead to advances in guided tissue regeneration. Micropatterning of $3 \mathrm{D}$ natural 
biopolymers has already been realized in Type I Collagen gels, originally described by Nelson et al. (Figure 5C) [147]. Simple stamping techniques were applied to these 3D gels to form reproducible arrays of rectangular cultures. This geometrical conformation of tissue has allowed for very interesting studies of how mechanotaxis and morphogen gradients regulate cell invasion in three dimensions [148, 149].

As discussed in the durotaxis and haptotaxis sections, the ability of cells to exert tractional forces on the surrounding matrix directly affects their ability to migrate. Measurements of cell tractional forces, either through the cytoskeleton, or the matrix itself, is critical in observing this exerted force. Traction force microscopy has been a powerful tool in the past two decades in observing these tractional dynamics (Figure 5B, for review, see [18]). Substrate deformations can be visualized by incorporating fluorescent beads in the hydrogel matrix. Displacement of the beads is translated to tractional forces using continuum mechanics [150]. Traction force microscopy has been used to calculate the ability of cells to sense and exert stress in two dimensions via PAA substrates [151-153], and to describe cancer mechanosensing [154, 155]. More recently, thin film arrays have been developed to measure force exertion by cells without the need for embedded beads [156]. Analogous to the widely published postarrays, the displacement of the thin films via cell-generated tension can be measured by unsophisticated optical microscopy [157].

As the number of interesting convoluting physicochemical parameters increases, one lingering limitation of biomaterials-based migration studies is the lack of highthroughput technology. The ability to screen chemotherapeutics in 96-well formats, to test a wide variety of variables and combinations at once, is common in pharmaceutical companies. This type of format has been recently adapted for migration studies, to quantify migration and signaling in response to growth factors and inhibitors [158]. Though not yet available, adapting this type of technology to a biomaterials system would allow one to study these combinatorial agents in an engineered environment with control of stiffness, mesh sizes, adhesive background, etc. 


\section{FUTURE OUTLOOK}

While in vivo studies have provided the importance of cell migration in the context of physiology and disease, in vitro studies have been critical in providing mechanistic insights into the migratory process. The cell biology community has indeed enriched our understanding of the molecular components and their interdependencies involved in generating motility. However, the particular complexity of the locomotive process discussed in this review calls for an integrative approach for a comprehensive and predictive understanding of cell migration. Studies of cell motility using biomaterials systems have demonstrated the power of natural and synthetic materials in contributing to the mechanistic regulation of relevant phenomenological parameters, such as cell speed and directional persistence. Unfortunately, synthetic biomaterials systems have not yet gained sufficient traction in the biosciences community, often due to a mismatch between the pertinent biological questions and the physiological relevance of the materials. With the recent efforts for fostering interdisciplinary work, we posit that this gap will soon be bridged. We urge the biomaterials community to assess the vast space of unanswered, biologically relevant questions that will only be answered with elegant synthetic techniques. In turn, the cell biologists can appreciate the immense opportunities provided by thoughtfully designed materials, wherein carefully controlled microenvironments enable reproducible studies of complex mechanisms. 


\section{FIGURE CAPTIONS}

Figure 1 - Cell migration processes in a three-dimensional extracellular matrix. In vivo, cell motility is governed by the coordination of multiple extracellular signals, including soluble growth factors, the presentation of insoluble adhesive proteins, and the stiffness and extent of crosslinking of the matrix, among others, across multiple length scales. Reviewed here, biomaterials present the unique opportunity to quantitatively parse each process for a better understanding of the migratory process in a complex microenvironment. Red arrows designate tunable parameters in an engineered biomaterial environment, with the resulting control over cell migration shown in italics. 
FIGURE 2

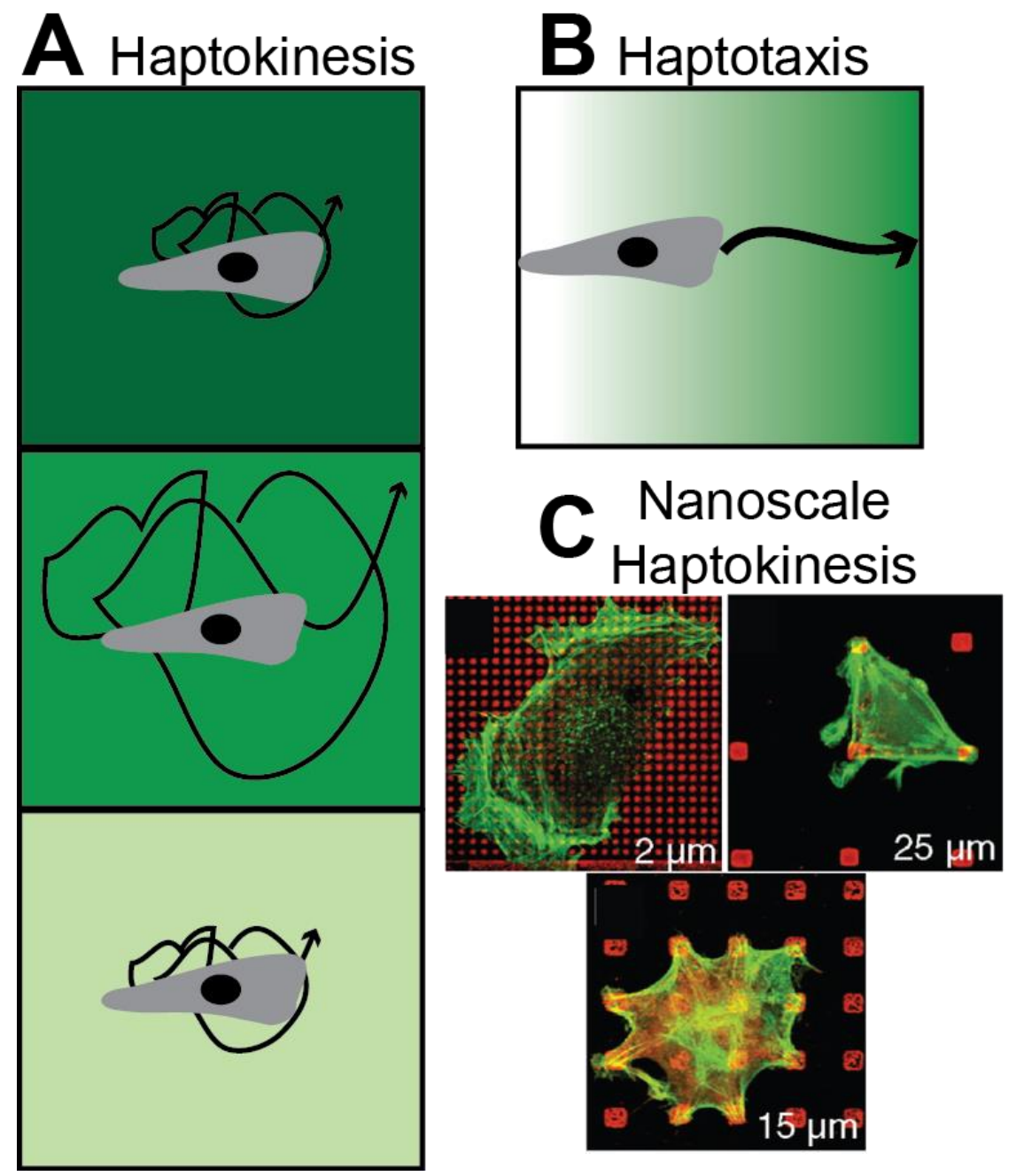

Figure 2: Adhesion-mediated cell migration. In (A), a homogeneous coating of surfaces with insoluble biomolecules at various densities (haptokinesis, shown by increasing background intensity) regulates cell motile speed in a biphasic fashion for many cell types and systems, analogously to chemokinesis. (B) Haptotaxis, on the other hand, is the directed guidance of cell migration by presenting cell adhesive molecules on a gradient, analogously to chemotaxis. (C) On the nanoscale, adhesive biomolecules can be spaced at defined intervals with soft lithography to determine the spatial requirements for focal adhesion formation to generate functional motility (permission from [79] pending). 


\section{FIGURE 3}
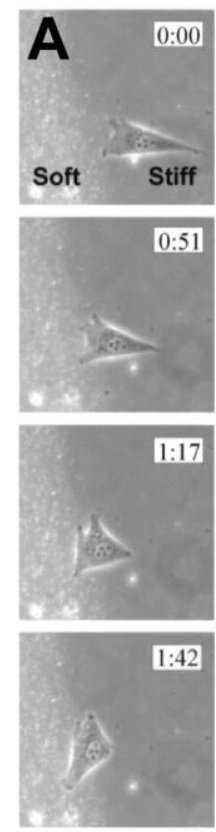

$1: 42$
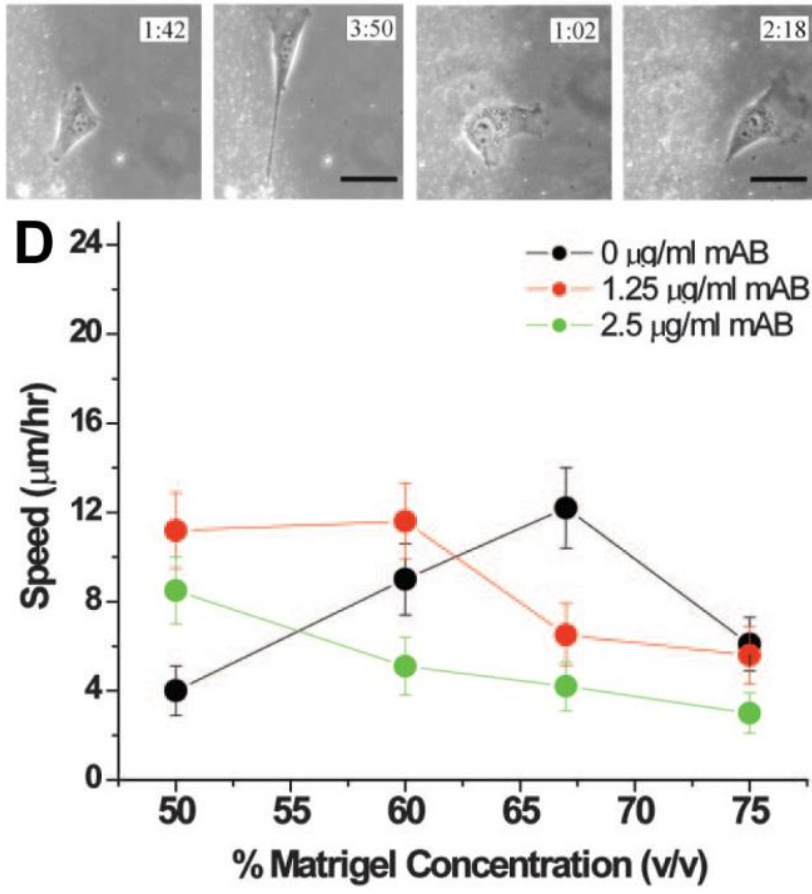

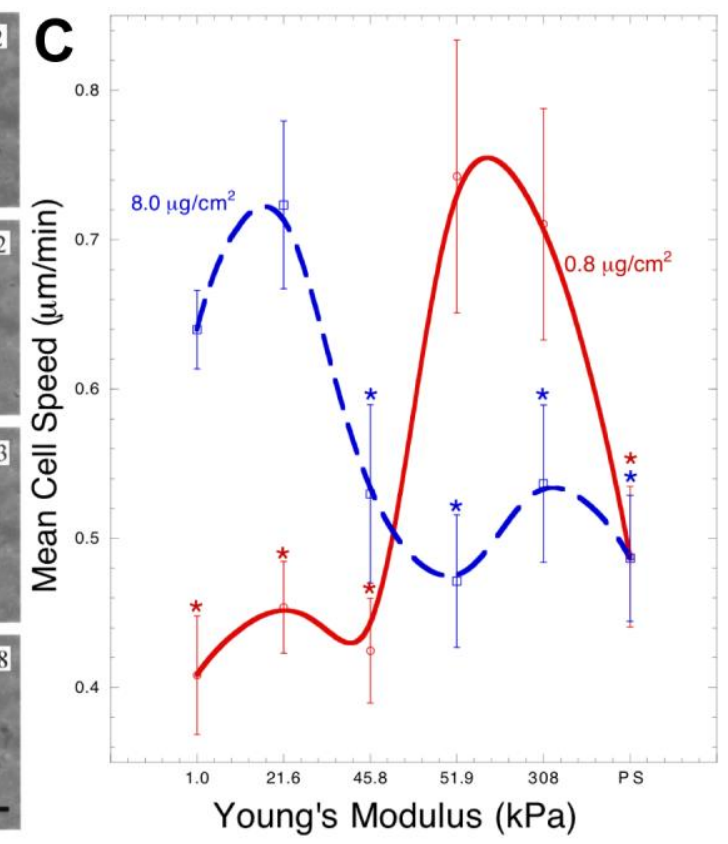

Figure 3: The stiffness of the matrix regulates cell motility. PAA substrates were used to show the first evidence that the stiffness of an underlying substratum could direct cell motility (A-B). Migrating cells starting on a stiff (A) or soft (B) substrate would come to an interface between soft and stiff substrates and preferentially migrate on stiffer substrates (permission from [89] pending). On PAA substrates spanning a range of stiffnesses, smooth muscle cells were shown to have a biphasic dependence on substrate stiffness $(C)$. Cell motility was maximized on substrates of intermediate 
stiffness, which further depended on the density of fibronectin coupled to the PAA surface (permission from [91] pending). This biphasic relationship between matrix stiffness and cell motility was later shown in prostate cancer cells in 3D Matrigel environments ( $D$, permission from [61] pending). 


\section{FIGURE 4}
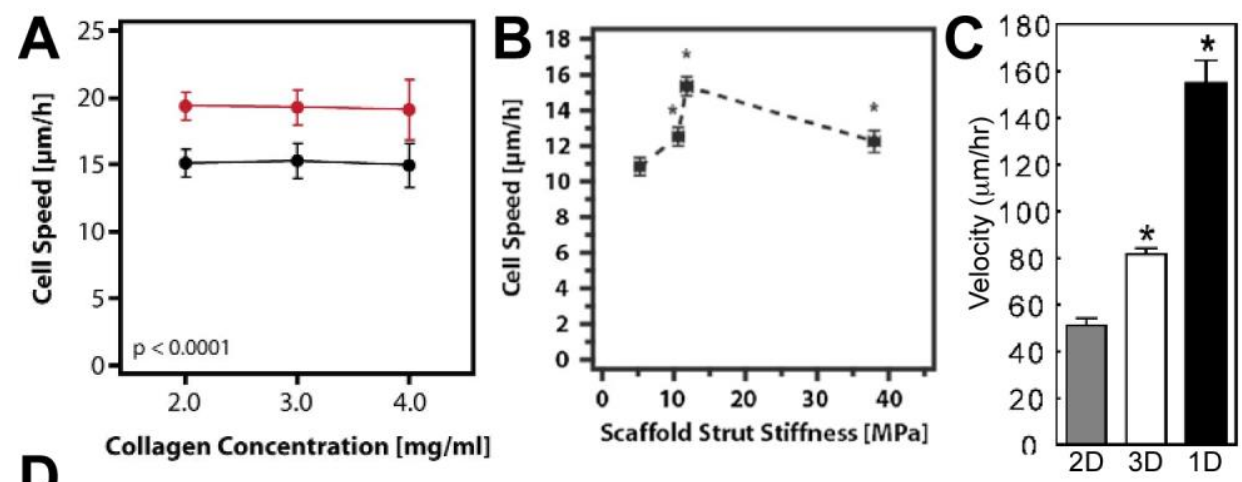

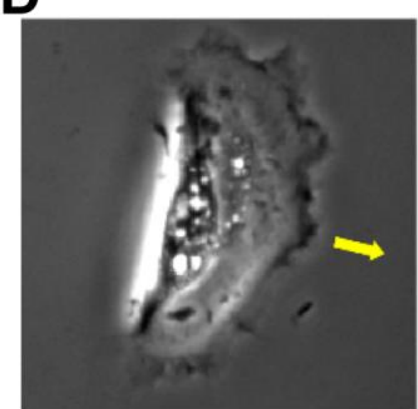

2D Surface

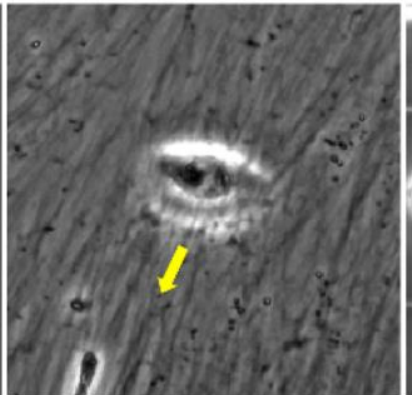

3D ECM

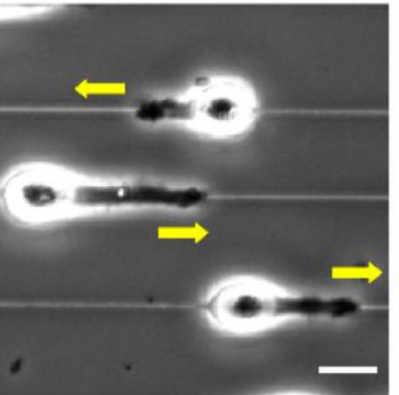

1D Lines

Figure 4: Fiber Length-Scale Dictates Geometric Migratory Microenvironment. In (A) Kim et al., observed no dependence of glioblastoma motility on 3D collagen gel stiffness (permission from [119] pending). In (B) Harley et. al., used collagen gels with pore sizes 1-2 orders of magnitude larger than in Kim et al. (permission from [132] pending). They observed cell speeds that had a biphasic dependence on collagen fiber stiffness. Pore sizes in this microenvironment are an order of magnitude larger than the length-scale of the cell, so collagen fibers appear as 1D lines. This may explain the discrepancy in observed motility between (A) and (B). In (C-D) Doyle et al., used micropatterned PDMS substrata and cell-generated 3D ECMs to show that cell migration is fastest along $1 \mathrm{D}$ printed lines and slowest on a non-fibrillar, flat 2D surface (permission from [133] pending). 


\section{FIGURE 5}

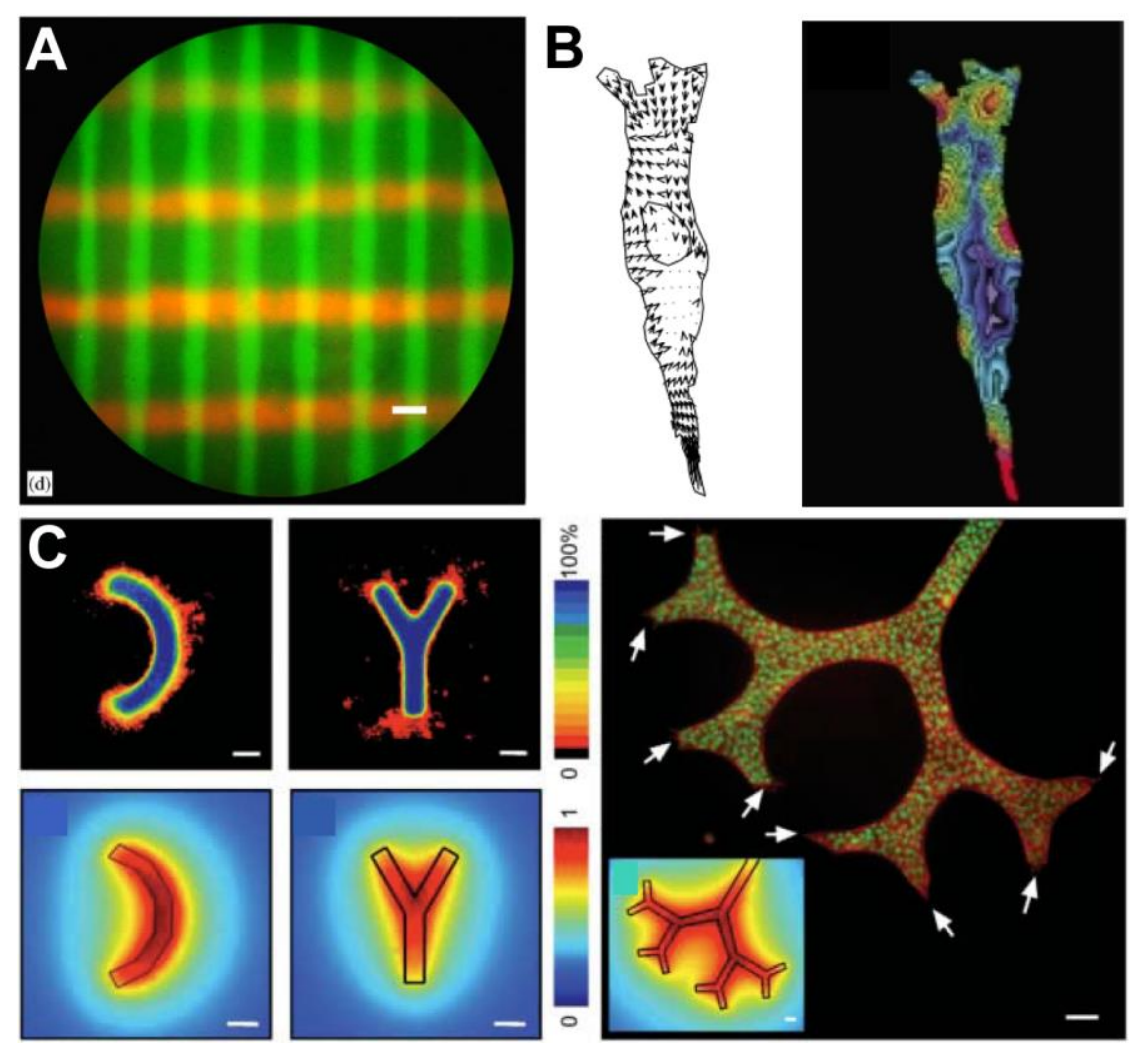

Figure 5: Emerging tools for studying matrix control of cell motility. The combination of light sensitive polymerization initiators and confocal microscopy has made 3D patterning of hydrogels possible (A, permission from [159] pending). Embedding and tracking the displacement of fluorescent beads in compliant substrates can be exploited to map tractional forces of migrating cells ( $B$, permission from [160] pending). Patterning in 3D matrices allows for formation of microtissues to study cell migration from tissue-like structures (C, permission from [147] pending). 


\section{REFERENCES}

1. Maschhoff, K.L. and H.S. Baldwin, Molecular determinants of neural crest migration. American Journal of Medical Genetics, 2000. 97(4): p. 280-288.

2. Keller, R., Cell migration during gastrulation. Current Opinion in Cell Biology, 2005. 17(5): p. 533-541.

3. Aman, A. and T. Piotrowski, Cell migration during morphogenesis. Developmental Biology, 2010. 341(1): p. 20-33.

4. Braiman-Wiksman, L., et al., Novel insights into wound healing sequence of events. Toxicologic Pathology, 2007. 35(6): p. 767-779.

5. Sánchez-Madrid, F. and M.A.d. Pozo, Leukocyte polarization in cell migration and immune interactions. The EMBO Journal, 1999. 18(3): p. 501-511.

6. Friedl, P. and B. Weigelin, Interstitial leukocyte migration and immune function. Nature Immunology, 2008. 9(9): p. 960-969.

7. Yang, J. and R.A. Weinberg, Epithelial-mesenchymal transition: at the crossroads of development and tumor metastasis. Dev Cell, 2008. 14(6): p. 818829.

8. Lauffenburger, D.A., Horwitz, A.F., Cell migration: a physically integrated molecular process. Cell, 1996. 84: p. 359-69.

9. Ridley, A.J., et al., Cell migration: integrating signals from front to back. Science, 2003. 302(5651): p. 1704-1709.

10. Machesky, L.M., Lamellipodia and filopodia in metastasis and invasion. FEBS Letters, 2008. 582(14): p. 2102-2111.

11. Webb, D.J., Parsons, T., Horwitz, A.F., Adhesion assembly, disassembly and turnover in migrating cells - over and over and over again. Nat Cell Biol, 2002. 4: p. E97-E100.

12. Wozniak, M.A., et al., Focal adhesion regulation of cell behavior. Biochim Biophys Acta, 2004. 1692(2-3): p. 103-19.

13. Etienne-Manneville, S., Polarity proteins in migration and invasion. Oncogene, 2008. 27(55): p. 6970-6980.

14. Etienne-Manneville, S. and A. Hall, Rho GTPases in cell biology. Nature, 2002. 420(6916): p. 629-35.

15. Hall, A., Rho GTPases and the actin cytoskeleton. Science, 1998. 279(5350): p. 509-14.

16. Pertz, O., et al., Spatiotemporal dynamics of RhoA activity in migrating cells. Nature, 2006. 440(7087): p. 1069-72.

17. Wittmann, T. and C.M. Waterman-Storer, Cell motility: can Rho GTPases and microtubules point the way? J Cell Sci, 2001. 114(Pt 21): p. 3795-803.

18. Mierke, C.T., et al., Contractile forces in tumor cell migration. European Journal of Cell Biology, 2008. 87(8-9): p. 669-676.

19. Glading, A., D.A. Lauffenburger, and A. Wells, Cutting to the chase: calpain proteases in cell motility. Trends in cell biology, 2002. 12(1): p. 46-54.

20. Lammermann, T., et al., Rapid leukocyte migration by integrin-independent flowing and squeezing. Nature, 2008. 453(7191): p. 51-5. 
21. Wolf, K., et al., Compensation mechanism in tumor cell migration: mesenchymalamoeboid transition after blocking of pericellular proteolysis. The Journal of Cell Biology, 2003. 160(2): p. 267-277.

22. Even-Ram, S. and K.M. Yamada, Cell migration in 3D matrix. Curr Opin Cell Biol, 2005. 17(5): p. 524-32.

23. Friedl, P. and K. Wolf, Tumour-cell invasion and migration: diversity and escape mechanisms. Nature Reviews. Cancer, 2003. 3(5): p. 362-374.

24. Wolf, K., et al., Amoeboid shape change and contact guidance: T-lymphocyte crawling through fibrillar collagen is independent of matrix remodeling by MMPs and other proteases. Blood, 2003. 102(9): p. 3262-3269.

25. Horssen, R.v. and T.L.M.t. Hagen, Crossing barriers: the new dimension of $2 D$ cell migration assays. J Cell Physiol, 2011. 226(1): p. 288-290.

26. Hubbell, J.A., Bioactive biomaterials. Curr Opin Biotechnol, 1999. 10(2): p. 123-9.

27. Locascio, A. and M.A. Nieto, Cell movements during vertebrate development: integrated tissue behaviour versus individual cell migration. Current Opinion in Genetics \\& Development, 2001. 11(4): p. 464-469.

28. Rutkowski, J.M. and M.A. Swartz, A driving force for change: interstitial flow as a morphoregulator. Trends Cell Biol, 2007. 17(1): p. 44-50.

29. Fleury, M.E., K.C. Boardman, and M.A. Swartz, Autologous morphogen gradients by subtle interstitial flow and matrix interactions. Biophys J, 2006. 91(1): p. 11321.

30. Helm, C.L., et al., Synergy between interstitial flow and VEGF directs capillary morphogenesis in vitro through a gradient amplification mechanism. Proc Natl Acad Sci U S A, 2005. 102(44): p. 15779-84.

31. Kedrin, D., et al., Cell motility and cytoskeletal regulation in invasion and metastasis. Journal of mammary gland biology and neoplasia, 2007. 12(2-3): p. $143-52$.

32. Condeelis, J. and J.W. Pollard, Macrophages: obligate partners for tumor cell migration, invasion, and metastasis. Cell, 2006. 124(2): p. 263-6.

33. Boyden, S., The chemotactic effect of mixtures of antibody and antigen on polymorphonuclear leucocytes. The Journal of experimental medicine, 1962. 115: p. 453-66.

34. Nelson, R.D., P.G. Quie, and R.L. Simmons, Chemotaxis under agarose: a new and simple method for measuring chemotaxis and spontaneous migration of human polymorphonuclear leukocytes and monocytes. Journal of immunology, 1975. 115(6): p. 1650-6.

35. Rothman, C. and D. Lauffenburger, Analysis of the linear under-agarose leukocyte chemotaxis assay. Ann Biomed Eng, 1983. 11(5): p. 451-77.

36. Ford, R.M., et al., Measurement of bacterial random motility and chemotaxis coefficients: I. Stopped-flow diffusion chamber assay. Biotechnol Bioeng, 1991. 37(7): p. 647-60.

37. Toetsch, S., et al., The evolution of chemotaxis assays from static models to physiologically relevant platforms. Integr Biol (Camb), 2009. 1(2): p. 170-81.

38. Cheng, S.Y., et al., A hydrogel-based microfluidic device for the studies of directed cell migration. Lab Chip, 2007. 7(6): p. 763-9. 
39. Chung, S., et al., Cell migration into scaffolds under co-culture conditions in a microfluidic platform. Lab Chip, 2009. 9(2): p. 269-75.

40. Saadi, W., et al., Generation of stable concentration gradients in $2 D$ and $3 D$ environments using a microfluidic ladder chamber. Biomed Microdevices, 2007. 9(5): p. 627-35.

41. Platt, M.O., et al., Sustained epidermal growth factor receptor levels and activation by tethered ligand binding enhances osteogenic differentiation of multipotent marrow stromal cells. J Cell Physiol, 2009. 221(2): p. 306-17.

42. Hubbell, J.A., Matrix-bound growth factors in tissue repair. Swiss Med Wkly, 2007. 137 Suppl 155: p. 72S-76S.

43. Fan, V.H., et al., Tethered epidermal growth factor provides a survival advantage to mesenchymal stem cells. Stem Cells, 2007. 25(5): p. 1241-51.

44. Stefonek-Puccinelli, T.J. and K.S. Masters, Co-immobilization of gradientpatterned growth factors for directed cell migration. Ann Biomed Eng, 2008. 36(12): p. 2121-33.

45. Philippar, U., et al., A Mena invasion isoform potentiates EGF-induced carcinoma cell invasion and metastasis. Developmental cell, 2008. 15(6): p. 813-28.

46. Ng, C.P. and M.A. Swartz, Fibroblast alignment under interstitial fluid flow using a novel 3-D tissue culture model. Am J Physiol Heart Circ Physiol, 2003. 284(5): p. $\mathrm{H} 1771-7$.

47. Geiger, B., et al., Transmembrane crosstalk between the extracellular matrix-cytoskeleton crosstalk. Nature Reviews. Molecular Cell Biology, 2001. 2(11): p. 793-805.

48. Alberts, B., Molecular biology of the cell. Book2002.

49. Kubow, K.E. and A.R. Horwitz, Reducing background fluorescence reveals adhesions in 3D matrices. Nat Cell Biol, 2011. 13(1): p. 3-5; author reply 5-7.

50. Cukierman, E., et al., Taking cell-matrix adhesions to the third dimension. Science, 2001. 294(5547): p. 1708-12.

51. Yousaf, M.N., Model substrates for studies of cell mobility. Current Opinion in Chemical Biology, 2009. 13(5-6): p. 697-704.

52. Seidi, A., et al., Gradient biomaterials for soft-to-hard interface tissue engineering. Acta Biomaterialia, 2011.

53. Palecek, S.P., et al., Integrin-ligand binding properties govern cell migration speed through cell-substratum adhesiveness. Nature, 1997. 385(6616): p. 53740.

54. DiMilla, P.A., et al., Maximal migration of human smooth muscle cells on fibronectin and type IV collagen occurs at an intermediate attachment strength. J Cell Biol, 1993. 122(3): p. 729-37.

55. Gupton, S.L. and C.M. Waterman-Storer, Spatiotemporal feedback between actomyosin and focal-adhesion systems optimizes rapid cell migration. Cell, 2006. 125(7): p. 1361-1374.

56. Maheshwari, G., et al., Cell adhesion and motility depend on nanoscale RGD clustering. J Cell Sci, 2000. 113 ( Pt 10): p. 1677-1686.

57. Wells, A., et al., Growth factor-induced cell motility in tumor invasion. Acta Oncologica (Stockholm, Sweden), 2002. 41(2): p. 124-130. 
58. Tashiro, K., et al., $A$ synthetic peptide containing the IKVAV sequence from the $A$ chain of laminin mediates cell attachment, migration, and neurite outgrowth. The Journal of Biological Chemistry, 1989. 264(27): p. 16174-16182.

59. Ruoslahti, E., RGD and other recognition sequences for integrins. Annu Rev Cell Dev Biol, 1996. 12: p. 697-715.

60. Wacker, B.K., et al., Endothelial cell migration on RGD-peptide-containing PEG hydrogels in the presence of sphingosine 1-phosphate. Biophys J, 2008. 94(1): p. 273-285.

61. Zaman, M.H., et al., Migration of tumor cells in $3 D$ matrices is governed by matrix stiffness along with cell-matrix adhesion and proteolysis. Proc Natl Acad Sci U S A, 2006. 103(29): p. 10889-94.

62. Mann, B.K. and J.L. West, Cell adhesion peptides alter smooth muscle cell adhesion, proliferation, migration, and matrix protein synthesis on modified surfaces and in polymer scaffolds. J Biomed Mater Res, 2002. 60(1): p. 86-93.

63. Schwartz, M.P., et al., A synthetic strategy for mimicking the extracellular matrix provides new insight about tumor cell migration. Integr Biol (Camb), 2010. 2(1): p. 32-40.

64. Petrie, R.J., A.D. Doyle, and K.M. Yamada, Random versus directionally persistent cell migration. Nat Rev Mol Cell Biol, 2009. 10(8): p. 538-49.

65. Ware, M.F., A. Wells, and D.A. Lauffenburger, Epidermal growth factor alters fibroblast migration speed and directional persistence reciprocally and in a matrix-dependent manner. J Cell Sci, 1998. 111 ( Pt 16): p. 2423-32.

66. Harms, B.D., et al., Directional persistence of EGF-induced cell migration is associated with stabilization of lamellipodial protrusions. Biophys J, 2005. 88(2): p. 1479-88.

67. Cavalcanti-Adam, E.A., et al., Cell spreading and focal adhesion dynamics are regulated by spacing of integrin ligands. Biophys J, 2007. 92(8): p. 2964-2974.

68. Glading, A., et al., Epidermal growth factor activates $m$-calpain (calpain II), at least in part, by extracellular signal-regulated kinase-mediated phosphorylation. Mol Cell Biol, 2004. 24(6): p. 2499-2512.

69. Takino, T., et al., Membrane-type 1 matrix metalloproteinase modulates focal adhesion stability and cell migration. Experimental Cell Research, 2006. 312(8): p. 1381-1389.

70. Arnold, M., et al., Induction of cell polarization and migration by a gradient of nanoscale variations in adhesive ligand spacing. Nano Letters, 2008. 8(7): p. 2063-2069.

71. Pulsipher, A. and M.N. Yousaf, Surface chemistry and cell biological tools for the analysis of cell adhesion and migration. Chembiochem: A European Journal of Chemical Biology, 2010. 11(6): p. 745-753, 730.

72. Gobin, A.S. and J.L. West, Effects of epidermal growth factor on fibroblast migration through biomimetic hydrogels. Biotechnology Progress, 2003. 19(6): p. 1781-1785.

73. Lamb, B.M., N.P. Westcott, and M.N. Yousaf, Microfluidic lithography to create dynamic gradient SAM surfaces for spatio-temporal control of directed cell migration. Chembiochem: A European Journal of Chemical Biology, 2008. 9(16): p. 2628-2632. 
74. Smith, J.T., J.T. Elkin, and W.M. Reichert, Directed cell migration on fibronectin gradients: effect of gradient slope. Experimental Cell Research, 2006. 312(13): p. 2424-2432.

75. Smith, J.T., et al., Measurement of cell migration on surface-bound fibronectin gradients. Langmuir : the ACS journal of surfaces and colloids, 2004. 20(19): $p$. 8279-8286.

76. Smith, J.T., D.H. Kim, and W.M. Reichert, Haptotactic gradients for directed cell migration: stimulation and inhibition using soluble factors. Combinatorial Chemistry \\& High Throughput Screening, 2009. 12(6): p. 598-603.

77. DeLong, S.A., A.S. Gobin, and J.L. West, Covalent immobilization of RGDS on hydrogel surfaces to direct cell alignment and migration. Journal of Controlled Release: Official Journal of the Controlled Release Society, 2005. 109(1-3): p. 139-148.

78. Guarnieri, D., et al., Covalently immobilized RGD gradient on PEG hydrogel scaffold influences cell migration parameters. Acta Biomaterialia, 2010. 6(7): p. 2532-2539.

79. Chan, E.W.L. and M.N. Yousaf, A photo-electroactive surface strategy for immobilizing ligands in patterns and gradients for studies of cell polarization. Mol Biosyst, 2008. 4(7): p. 746-753.

80. Lee, E.-J., E.W.L. Chan, and M.N. Yousaf, Spatio-temporal control of cell coculture interactions on surfaces. Chembiochem: A European Journal of Chemical Biology, 2009. 10(10): p. 1648-1653.

81. Lamb, B.M., S. Park, and M.N. Yousaf, Microfluidic permeation printing of selfassembled monolayer gradients on surfaces for chemoselective ligand immobilization applied to cell adhesion and polarization. Langmuir: The ACS Journal of Surfaces and Colloids, 2010. 26(15): p. 12817-12823.

82. Califano, J.P. and C.A. Reinhart-King, Exogenous and endogenous force regulation of endothelial cell behavior. J Biomech. 43(1): p. 79-86.

83. Engler, A.J., et al., Matrix elasticity directs stem cell lineage specification. Cell, 2006. 126(4): p. 677-89.

84. Engler, A.J., et al., Myotubes differentiate optimally on substrates with tissue-like stiffness: pathological implications for soft or stiff microenvironments. J Cell Biol, 2004. 166(6): p. 877-87.

85. Peyton, S.R., et al., The effects of matrix stiffness and RhoA on the phenotypic plasticity of smooth muscle cells in a 3-D biosynthetic hydrogel system.

Biomaterials, 2008. 29(17): p. 2597-607.

86. Peyton, S.R., et al., The use of poly(ethylene glycol) hydrogels to investigate the impact of ECM chemistry and mechanics on smooth muscle cells. Biomaterials, 2006. 27(28): p. 4881-93.

87. Khatiwala, C.B., et al., ECM compliance regulates osteogenesis by influencing MAPK signaling downstream of RhoA and ROCK. J Bone Miner Res, 2009. 24(5): p. 886-98.

88. Peyton, S.R., et al., The emergence of ECM mechanics and cytoskeletal tension as important regulators of cell function. Cell Biochem Biophys, 2007. 47(2): $\mathrm{p}$. 300-20. 
89. Lo, C.M., et al., Cell movement is guided by the rigidity of the substrate. Biophys J, 2000. 79(1): p. 144-52.

90. Thompson, M.T., et al., Biochemical functionalization of polymeric cell substrata can alter mechanical compliance. Biomacromolecules, 2006. 7(6): p. 1990-1995.

91. Peyton, S.R. and A.J. Putnam, Extracellular matrix rigidity governs smooth muscle cell motility in a biphasic fashion. J Cell Physiol, 2005. 204(1): p. 198209.

92. Khatiwala, C.B., S.R. Peyton, and A.J. Putnam, Intrinsic mechanical properties of the extracellular matrix affect the behavior of pre-osteoblastic MC3T3-E1 cells. Am J Physiol Cell Physiol, 2006. 290(6): p. C1640-50.

93. Stroka, K.M. and H. Aranda-Espinoza, Neutrophils display biphasic relationship between migration and substrate stiffness. Cell Motil Cytoskeleton, 2009. 66(6): p. 328-41.

94. Ulrich, T.A., E.M. de Juan Pardo, and S. Kumar, The mechanical rigidity of the extracellular matrix regulates the structure, motility, and proliferation of glioma cells. Cancer Res, 2009. 69(10): p. 4167-74.

95. Wong, J.Y., et al., Directed movement of vascular smooth muscle cells on gradient-compliant hydrogels. Langmuir, 2003. 19(5): p. 1908-1913.

96. Burdick, J.A., A. Khademhosseini, and R. Langer, Fabrication of gradient hydrogels using a microfluidics/photopolymerization process. Langmuir, 2004. 20(13): p. 5153-5156.

97. Zaari, N., et al., Photopolymerization in microfluidic gradient generators: Microscale control of substrate compliance to manipulate cell response. Advanced Materials, 2004. 16(23-24): p. 2133-+.

98. Isenberg, B.C., et al., Vascular Smooth Muscle Cell Durotaxis Depends on Substrate Stiffness Gradient Strength. Biophysical Journal, 2009. 97(5): p. 13131322.

99. Grinnell, F. and W.M. Petroll, Cell Motility and Mechanics in Three-Dimensional Collagen Matrices. Annu Rev Cell Dev Biol.

100. Miron-Mendoza, M., J. Seemann, and F. Grinnell, The differential regulation of cell motile activity through matrix stiffness and porosity in three dimensional collagen matrices. Biomaterials, 2010. 31(25): p. 6425-6435.

101. Zaman, M.H., et al., Computational model for cell migration in three-dimensional matrices. Biophys J, 2005. 89(2): p. 1389-97.

102. Baker, E.L. and M.H. Zaman, The biomechanical integrin. J Biomech. 43(1): p. 38-44.

103. Shih, J. and R. Keller, Patterns of cell motility in the organizer and dorsal mesoderm of Xenopus laevis. Development, 1992. 116(4): p. 915-30.

104. Chien, S., et al., Molecular basis of mechanical modulation of endothelial cell migration. Front Biosci, 2005. 10: p. 1985-2000.

105. Shemesh, T., et al., Focal adhesions as mechanosensors: a physical mechanism. Proc Natl Acad Sci U S A, 2005. 102(35): p. 12383-8.

106. Geiger, B. and A. Bershadsky, Exploring the neighborhood: adhesion-coupled cell mechanosensors. Cell, 2002. 110(2): p. 139-42.

107. Mitchison, T. and M. Kirschner, Cytoskeletal dynamics and nerve growth. Neuron, 1988. 1(9): p. 761-72. 
108. Chan, C.E. and D.J. Odde, Traction dynamics of filopodia on compliant substrates. Science, 2008. 322(5908): p. 1687-91.

109. Stachowiak, A.N., et al., Bioactive hydrogels with an ordered cellular structure combine interconnected macroporosity and robust mechanical properties. Advanced Materials, 2005. 17(4): p. 399-403.

110. Nemir, S., H.N. Hayenga, and J.L. West, PEGDA hydrogels with patterned elasticity: Novel tools for the study of cell response to substrate rigidity. Biotechnol Bioeng. 105(3): p. 636-44.

111. Griffith, L.G. and M.A. Swartz, Capturing complex 3D tissue physiology in vitro. Nat Rev Mol Cell Biol, 2006. 7(3): p. 211-24.

112. Smalley, K.S., M. Lioni, and M. Herlyn, Life isn't flat: taking cancer biology to the next dimension. In Vitro Cell Dev Biol Anim, 2006. 42(8-9): p. $242-7$.

113. Stamenkovic, I., Matrix metalloproteinases in tumor invasion and metastasis. Seminars in Cancer Biology, 2000. 10(6): p. 415-433.

114. Friedl, P. and K. Wolf, Proteolytic interstitial cell migration: a five-step process. Cancer metastasis reviews, 2009. 28(1-2): p. 129-135.

115. Wolf, K. and P. Friedl, Molecular mechanisms of cancer cell invasion and plasticity. The British Journal of Dermatology, 2006. 154 Suppl 1: p. 11-15.

116. Zaman, M.H., P. Matsudaira, and D.A. Lauffenburger, Understanding effects of matrix protease and matrix organization on directional persistence and translational speed in three-dimensional cell migration. Ann Biomed Eng, 2007. 35(1): p. 91-100.

117. Burgess, B.T., J.L. Myles, and R.B. Dickinson, Quantitative analysis of adhesionmediated cell migration in three-dimensional gels of RGD-grafted collagen. Ann Biomed Eng, 2000. 28(1): p. 110-118.

118. Raeber, G.P., M.P. Lutolf, and J.A. Hubbell, Molecularly engineered PEG hydrogels: a novel model system for proteolytically mediated cell migration. Biophys J, 2005. 89(2): p. 1374-88.

119. Kim, H.-D., et al., Epidermal Growth Factor-induced Enhancement of Glioblastoma Cell Migration in 3D Arises from an Intrinsic Increase in Speed But an Extrinsic Matrix- and Proteolysis-dependent Increase in Persistence. Mol. Biol. Cell, 2008. 19(10): p. 4249-4259.

120. Saffarian, S., et al., Interstitial collagenase is a Brownian ratchet driven by proteolysis of collagen. Science, 2004. 306(5693): p. 108-111.

121. Ghajar, C.M., et al., A novel three-dimensional model to quantify metastatic melanoma invasion. Mol Cancer Ther, 2007. 6(2): p. 552-61.

122. Lutolf, M.P. and J.A. Hubbell, Synthetic biomaterials as instructive extracellular microenvironments for morphogenesis in tissue engineering. Nat Biotechnol, 2005. 23(1): p. 47-55.

123. Raeber, G.P., M.P. Lutolf, and J.A. Hubbell, Mechanisms of 3-D migration and matrix remodeling of fibroblasts within artificial ECMs. Acta Biomater, 2007. 3(5): p. 615-629.

124. Miller, J.S., et al., Bioactive hydrogels made from step-growth derived PEGpeptide macromers. Biomaterials, 2010. 31(13): p. 3736-3743. 
125. Lee, S.-H., et al., Poly(ethylene glycol) hydrogels conjugated with a collagenasesensitive fluorogenic substrate to visualize collagenase activity during threedimensional cell migration. Biomaterials, 2007. 28(20): p. 3163-3170.

126. Galler, K.M., et al., Self-assembling multidomain peptide hydrogels: designed susceptibility to enzymatic cleavage allows enhanced cell migration and spreading. J Am Chem Soc, 2010. 132(9): p. 3217-3223.

127. Coussens, L.M., B. Fingleton, and L.M. Matrisian, Matrix metalloproteinase inhibitors and cancer: trials and tribulations. Science, 2002. 295(5564): p. 23872392.

128. Sabeh, F., R. Shimizu-Hirota, and S.J. Weiss, Protease-dependent versus independent cancer cell invasion programs: three-dimensional amoeboid movement revisited. The Journal of Cell Biology, 2009. 185(1): p. 11-19.

129. Friedl, P., K.S. Zanker, and E.B. Brocker, Cell migration strategies in 3-D extracellular matrix: differences in morphology, cell matrix interactions, and integrin function. Microsc Res Tech, 1998. 43(5): p. 369-78.

130. Martins, G.G. and J. Kolega, Endothelial cell protrusion and migration in threedimensional collagen matrices. Cell Motil Cytoskeleton, 2006. 63(2): p. 101-15.

131. Fraley, S.I., et al., A distinctive role for focal adhesion proteins in threedimensional cell motility. Nat Cell Biol.

132. Harley, B.A., et al., Microarchitecture of three-dimensional scaffolds influences cell migration behavior via junction interactions. Biophys J, 2008. 95(8): p. 401324.

133. Doyle, A.D., et al., One-dimensional topography underlies three-dimensional fibrillar cell migration. J Cell Biol, 2009. 184(4): p. 481-90.

134. Liu, Y., et al., Control of cell migration in two and three dimensions using substrate morphology. Exp Cell Res, 2009. 315(15): p. 2544-57.

135. Ochsner, M., et al., Dimensionality controls cytoskeleton assembly and metabolism of fibroblast cells in response to rigidity and shape. PLoS One, 2010. 5(3): p. e9445.

136. da Silva, J., et al., The cavity-to-cavity migration of leukaemic cells through $3 D$ honey-combed hydrogels with adjustable internal dimension and stiffness. Biomaterials, 2010. 31(8): p. 2201-2208.

137. Stachowiak, A.N., et al., Bioactive hydrogels with an ordered cellular structure combine interconnected macroporosity and robust mechanical properties. Advanced Materials, 2005. 17(4): p. 399-+.

138. Stachowiak, A.N. and D.J. Irvine, Inverse opal hydrogel-collagen composite scaffolds as a supportive microenvironment for immune cell migration. Journal of Biomedical Materials Research Part A, 2008. 85A(3): p. 815-828.

139. Peyton, S.R., et al., Marrow-Derived stem cell motility in 3D synthetic scaffold is governed by geometry along with adhesivity and stiffness. Biotechnol Bioeng, 2011.

140. Martins, A., et al., Electrospun nanostructured scaffolds for tissue engineering applications. Nanomedicine (Lond), 2007. 2(6): p. 929-42.

141. Mi, K., et al., Influence of a self-assembling peptide, RADA16, compared with collagen I and Matrigel on the malignant phenotype of human breast-cancer cells in 3D cultures and in vivo. Macromol Biosci, 2009. 9(5): p. 437-43. 
142. Horii, A., et al., Biological designer self-assembling peptide nanofiber scaffolds significantly enhance osteoblast proliferation, differentiation and 3-D migration. PLoS One, 2007. 2(2): p. e190.

143. Park, J., et al., TiO2 Nanotube Surfaces: $15 \mathrm{~nm}$ - An Optimal Length Scale of Surface Topography for Cell Adhesion and Differentiation. Small, 2009. 5(6): p. 666-671.

144. Brammer, K.S., et al., Enhanced cellular mobility guided by TiO2 nanotube surfaces. Nano Lett, 2008. 8(3): p. 786-93.

145. Sengupta, D. and S.C. Heilshorn, Protein-Engineered Biomaterials: Highly Tunable Tissue Engineering Scaffolds. Tissue Engineering Part B-Reviews, 2010. 16(3): p. 285-293.

146. Lee, S.H., J.J. Moon, and J.L. West, Three-dimensional micropatterning of bioactive hydrogels via two-photon laser scanning photolithography for guided 3D cell migration. Biomaterials, 2008. 29(20): p. 2962-8.

147. Nelson, C.M., et al., Tissue geometry determines sites of mammary branching morphogenesis in organotypic cultures. Science, 2006. 314(5797): p. 298-300.

148. Gjorevski, N. and C.M. Nelson, Endogenous patterns of mechanical stress are required for branching morphogenesis. Integr Biol (Camb). 2(9): p. 424-34.

149. Pavlovich, A.L., S. Manivannan, and C.M. Nelson, Adipose Stroma Induces Branching Morphogenesis of Engineered Epithelial Tubules. Tissue Eng Part A.

150. Pelham, R.J., Jr. and Y. Wang, Cell locomotion and focal adhesions are regulated by substrate flexibility. Proc Natl Acad Sci U S A, 1997. 94(25): p. 13661-5.

151. Sabass, B., et al., High resolution traction force microscopy based on experimental and computational advances. Biophys J, 2008. 94(1): p. 207-20.

152. Sen, S., A.J. Engler, and D.E. Discher, Matrix Strains Induced by Cells: Computing How Far Cells Can Feel. Cellular and Molecular Bioengineering, 2009. 2(1): p. 39-48.

153. Califano, J.P. and C.A. Reinhart-King, Exogenous and endogenous force regulation of endothelial cell behavior. Journal of Biomechanics, 2010. 43(1): p. 79-86.

154. Mierke, C.T., et al., Contractile forces in tumor cell migration. Eur J Cell Biol, 2008. 87(8-9): p. 669-76.

155. Raupach, C., et al., Stress fluctuations and motion of cytoskeletal-bound markers. Phys Rev E Stat Nonlin Soft Matter Phys, 2007. 76(1 Pt 1): p. 011918.

156. Zimberlin, J.A., P. Wadsworth, and A.J. Crosby, Living microlens arrays. Cell Motil Cytoskeleton, 2008. 65(9): p. 762-7.

157. Miquelard-Garnier, G., et al., Polymer microlenses for quantifying cell sheet mechanics. Soft Matter, 2010. 6(2): p. 398-403.

158. Joslin, E.J., et al., EGF-receptor-mediated mammary epithelial cell migration is driven by sustained ERK signaling from autocrine stimulation. J Cell Sci, 2007. 120(Pt 20): p. 3688-99.

159. Hahn, M.S., et al., Photolithographic patterning of polyethylene glycol hydrogels. Biomaterials, 2006. 27(12): p. 2519-24.

160. Beningo, K.A. and Y.L. Wang, Flexible substrata for the detection of cellular traction forces. Trends Cell Biol, 2002. 12(2): p. 79-84. 
